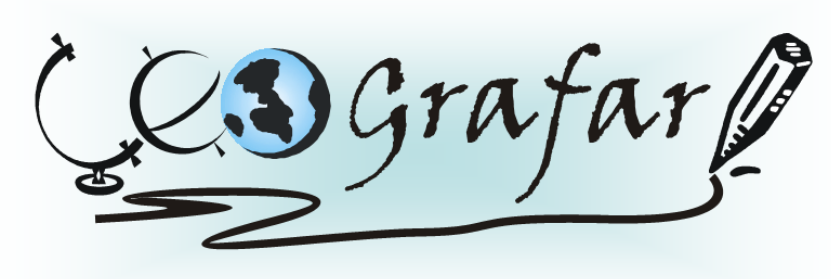

Revista Eletrônica do Programa de Pós-Graduação em Geografia - UFPR

\title{
DESARROLLO LOCAL Y ORDENAMIENTO TERRITORIAL EN LA PROVINCIA DE BUENOS AIRES
}

\author{
LICIA RÍOS
}

MARÍA JULIA ROCCA

\author{
NÉSTOR OMAR BONO
}

\begin{abstract}
Resumen: El territorio expresa la síntesis de la complejidad social, económica y política del proceso de desarrollo de una sociedad por lo que su ordenamiento debe considerarse como una política transversal a todas las acciones que buscan el desarrollo. Esta visión integral no fue la que siempre asumieron los enfoques de las políticas de desarrollo para los espacios locales, como tampoco las prácticas, que recorrieron un camino que, tanto en relación a los procedimientos como en las valoraciones del territorio, transitó periodos en los que se reconocen diferenciales enfoques. En este trabajo se analizan las políticas de ordenamiento territorial y su relación con las políticas de desarrollo, en municipios pequeños y medianos de la Provincia de Buenos Aires desde fines de la década del $70^{2}$, teniendo como hipótesis que la negación de este rol integrador y transversal ha minimizado las posibilidades del desarrollo de los mismos.
\end{abstract}

Palabras Clave: Desarrollo local; Ordenamiento territorial; Municipios pequeños y medianos

\section{DESENVOLVIMENTO LOCAL E ORDENAÇÃO TERRITORIAL NA PROVÍNCIA DE BUENOS AIRES}

Resumo: O território sintetiza o complexo social, econômico e político do processo de desenvolvimento de uma sociedade, de modo que a política de ordenamento territorial deve ser considerada como um projeto transversal a todas as ações que visam o desenvolvimento. Políticas locais, nem sempre teve essa visão, reconhecendo as diferentes abordagens nas últimas décadas. Este artigo analisa as políticas de ordenamento do território e sua relação com as políticas de desenvolvimento dos pequenos e médios municípios da Província de Buenos Aires desde o final da década de 70 , com a suposição de que a negação do papel integradora e inter-sistema tem minimizado o potencial de desenvolvimento.

Palavras-chave: Desenvolvimento local; Ordenação Territorial; Pequenos e médios municípios

\footnotetext{
${ }^{1}$ licia.rios@hotmail.com

${ }^{2}$ En 1977 se sancionó del decreto de la Ley $\mathrm{N}^{\mathrm{a}}$ 8912, instrumento normativo que rige el ordenamiento del territorio de la provincia, y regula el uso, apropiación, subdivisión y equipamiento del suelo; junto con la Ley Orgánica de las Municipalidades de la Pcia. De Buenos Aires (decreto Ley No 6769/58 y modificatorias).
} 


\section{PRESENTACIÓN}

La ponencia se desprende de un trabajo que indaga en posibles cambios en las Políticas de Ordenamiento en municipios pequeños y medianos, valorizándolas por su transversalidad en el conjunto de acciones que buscan el desarrollo de los ámbitos locales en favor de aprovechar el potencial de los territorios.

Esta visión integral no siempre estuvo presente en las políticas de desarrollo y ordenamiento de los espacios locales. Ambas recorrieron un camino que, tanto en relación a los procedimientos como en las valoraciones del territorio, transitó periodos en los que se alternan signos de confluencia, contraposición, negación y recorridos paralelos, por motivos diversos.

Si bien en la provincia de Buenos Aires los municipios tienen responsabilidades en materia de desarrollo y ordenamiento y respecto de su articulación, establecidas en la Carta Orgánica de las Municipalidades (Decreto Ley 6769/58) y en la Ley de Ordenamiento Territorial y Usos del Suelo ${ }^{3}$ de 1977 (Decreto Ley 8912), no siempre pueden hacer frente a los compromisos, debido a que poseen capacidades limitadas para formular e implementar políticas, incluso en casos que cuentan con importantes ventajas iniciales, para el desarrollo o que reciben ayudas exógenas de recursos productivos que pudieran posibilitar el progreso integral.

En los municipios pequeños y medianos de Argentina, aún hoy esta articulación resulta dificultosa, pues si bien la distribución poblacional no es determinante, al condicionar e implicar diseños institucionales mínimos, escasa capacidad financiera y de gasto, insuficiente disponibilidad de recursos (humanos y materiales) puede afirmarse que en términos generales condiciona las capacidades de los municipios al restringir fuertemente sus acciones ${ }^{4}$.

\footnotetext{
${ }^{3}$ Rige sobre la totalidad del territorio provincial, en materia de ordenamiento territorial, uso, subdivisión y ocupación del suelo, y establece entre otros aspectos las responsabilidades que le caben a los municipios en la formulación, implementación y evaluación del ordenamiento territorial en sus jurisdicciones.

${ }^{4}$ Los recursos de los partidos de la provincia tienen un doble origen, los originados por la propia jurisdicción y aquellos provenientes de la coparticipación provincial, cuya asignación, en gran porcentaje, está relacionada con el tamaño poblacional y la complejidad de los municipios. Esto implica para los municipios de mayor tamaño una concentración de ingresos que les permite contar con estructuras organizativas desarrolladas, que posibilitan elaborar y cobrar tasas, financiar a especialistas, o tener los cuerpos técnicos necesarios para formular políticas e implementarlas; mientras que los pequeños municipios comúnmente deben concentrar sus erogaciones en el pago de salarios y servicios y realizar limitadas inversiones.
} 
Especial atención merece la dispar composición, en cuanto a tamaño poblacional entre municipios metropolitanos y pampeanos de la provincia de Buenos Aires, donde si bien en conjunto representan un tercio de la población del país, en mayor número no superan los treinta mil habitantes ${ }^{5}$ (Min. de Gobierno de la Pcia. de Bs. As., 2007). Esta desigual composición puede observarse en la Figura 1, donde se observa una mayor participación en el interior pampeano de municipios pequeños (con menos de 20 mil habitantes) y medianos (entre 20.001 y 100 mil habitantes).

Particularmente en lo referido al ordenamiento, a más de 30 años de la sanción del Decreto Ley 8912, la mayoría de los municipios provinciales ha recorrido mínimamente el proceso de ordenamiento territorial fijado por la misma, e incluso se encuentran casos que exceden la posibilidad de encuadrarlos en reglas generales de ordenamiento (Garay S., 2005). $\mathrm{Y}$ tampoco se han definido las estrategias a nivel provincial y regional para el sector, que orienten a las políticas municipales.

En este marco, la investigación busca construir aportes teóricos y metodológicos que contribuyan al debate sobre la operativización del desarrollo local en los citados municipios pequeños y medianos ${ }^{6}$ a partir de indagar en el rol del ordenamiento territorial en este proceso.

\section{OBJETIVOS Y METODOLOGÍA}

Como avance en esta ponencia se indagarán las principales estrategias de desarrollo local y ordenamiento aplicadas en la provincia de Bs. As. desde la sanción del Decreto Ley

\footnotetext{
5 El conjunto de municipios metropolitanos (40 partidos) ocupa un 4,5\% de la superficie provincial y contiene al $75 \%$ de la población, mientras que los pertenecientes al espacio pampeano (94 partidos) representan el $95,5 \%$ de la superficie provincial y cuenta con el $25 \%$ restante de la población (Min. de Gobierno de la Pcia. de Bs. As., 2007).

$6 \quad$ Si bien os municipios pequeños y medianos en la Provincia de Bs. As. presentan características diversas por su ubicación geográfica, liderazgos de los equipos y características de las sociedades locales, puede afirmarse que presentan un conjunto de problemáticas comunes, que pueden resumirse en bajas oportunidades para el desarrollo local, vinculadas a escasez de inversiones públicas y privadas, insuficientes programas de promoción de las actividades económicas y de intercambio de la producción local, debilidad de las organizaciones locales, falta de oportunidades relacionadas con lo educativo y cultural, entre otros aspectos. En lo que respecta a las estructuras institucionales, generalmente son débiles para encargarse de la coordinación dentro de su territorio de las políticas de los distintos niveles del estado y para emprender acciones entre municipios vecinos.
} 
8912, momento que coincide con la imposición del modelo neoliberal, inductor (por necesidad) de los primeros impulsos del desarrollo local, hasta la actualidad.

Se trata de construir una visión panorámica de las políticas de desarrollo y ordenamiento desde fines de la década del '70 hasta comienzos del siglo XXI, indagando en los enfoques, estrategias e instrumentos, y particularmente en las relaciones entre desarrollo y ordenamiento. Es decir, se busca confrontar la lógica de la práctica política con las discusiones sobre desarrollo y ordenamiento. Finalmente se pretende enunciar las cuestiones de las políticas de ordenamiento indispensables de considerar para el desarrollo.

FIGURA1:MUNICIPIOS PEQUEÑOS Y MEDIANOS DEL INTERIOR PAMPEANO DE LA PROVINCIA DE BUENOS AIRES.
FIGURA 2: TRANSVERSALIDAD DEL ORDENAMIENTO TERRITORIAL EN EL DESARROLLO LOCAL.
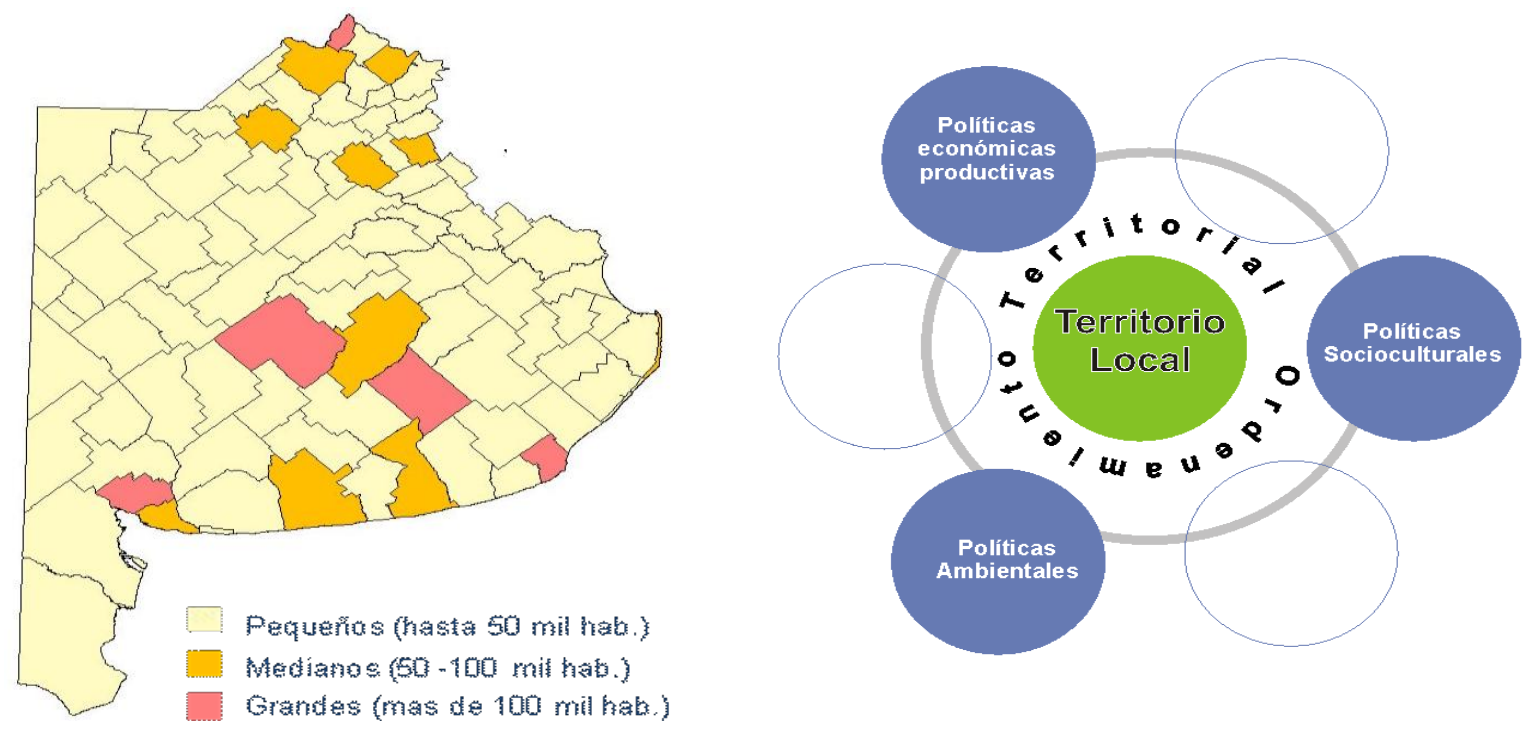

Fuente: Ríos y Rocca, Op. Cit.

La hipótesis que guía esta presentación es que las políticas de desarrollo local han visto minimizados sus resultados porque no se ha considerado este rol integrador y transversal del ordenamiento territorial (Fig. 2), aunque sí se ha logrado incorporar cierta valoración de los territorios para el desarrollo (Ríos y Rocca, 2007).

Persiguiendo el objetivo enunciado, se realizaron entrevistas a informantes calificados de organismos de la administración pública que han desempeñado funciones durante el 
periodo de análisis, se revisaron documentos públicos, estudios de casos y documentos de investigación.

Como se expresó anteriormente esta indagación empírica ha sido observada a partir de la construcción teórico conceptual en la que el territorio se construye más allá de lo espacial, incluyendo la dimensiones socioeconómica, cultural, y lo político, y en la que el desarrollo territorial como objetivo, hace referencia a un mejoramiento integral de la calidad de vida de la población. De aquí que en el ámbito local, las políticas de ordenamiento adquieren un rol estratégico y articulador en el desarrollo.

Por tratarse de una investigación en inicios, y por la necesaria síntesis para establecer relaciones entre procesos contemporáneos y de distintos periodos históricos, se asume que puede incurrirse en omisiones o imprecisiones, por lo que esta posibilidad de exponer los resultados obtenidos hasta la fecha resulta un espacio de apertura necesario y reconocido.

\section{ENFOQUES SOBRE EL DESARROLLO y ORDENAMIENTO}

A partir de la posguerra, el concepto de desarrollo se visualiza con la posibilidad de disfrute de seguridad económica y social, conservando hasta la década del 70 una directa relación entre desarrollo y crecimiento económico, momento a partir del cual se enfatiza en garantizar los beneficios sociales del desarrollo en términos de equidad. Con la consideración de las problemáticas ambientales se incorpora también la dimensión ecológica para integrarla con lo social y lo económico desde el concepto de desarrollo sustentable.

Años después, el Programa de las Naciones Unidas para el Desarrollo introduce una nueva acepción a partir del desarrollo humano, que puede describirse como proceso de ampliación de las opciones de la población, valorando beneficios que son menos materiales, así como la necesidad de un proyecto personal, la cohesión social y el derecho a afirmar sus tradiciones y cultura propia. (Boisier, 2001)

Cada una de estas concepciones del desarrollo incorpora nuevas dimensiones, en las que se enfatiza las necesidades a atender (social, económica, cultural, ecológica y política), ampliando la concepción de desarrollo, con el propósito de afrontar el desafío de conducir hacia el mejoramiento de la calidad de vida de una sociedad. 
Los enfoques más recientes incorporan la dimensión política a la noción de desarrollo, enfatizando en la construcción social (Boisier, Op. Cit.), en la promoción y fortalecimiento de confianzas y sentidos colectivos y entendiendo a las personas no como un recurso más, sino en cuanto a sus capacidades para definir su proyecto de desarrollo.

Estos contenidos incorporados en la noción de desarrollo, coexisten actualmente en diferentes enfoques, y en casos tienden a integrarse.

Paralelamente, estos cambios en la forma de entender al desarrollo, implican también evoluciones en cuanto a los actores involucrados y a los ámbitos de construcción de las políticas.

Inicialmente, con una clara orientación "estado- céntrica", los sujetos dominantes del desarrollo eran los actores de la sociedad nacional (burocracia estatal, elites modernizantes, vanguardias iluminadas, actores privados corporativos). Esta matriz de pensamiento, con una pretensión normativa universal (Kozlarek, 2001) construye "recetas" teóricamente aplicables a diferentes tiempos y lugares.

En las dos últimas décadas, estos roles son modificados, centrando ahora la decisión sobre la acumulación del capital y la asignación de recursos en los mercados internacionales, reconociéndose sólo a los actores globales y al mercado, como única dimensión relevante en los procesos de crecimiento, en desmedro del territorio local y sus actores.

Recientemente, desde una visión holística y constructiva del desarrollo (Boisier, 2003) se aproxima a la realidad admitiendo la diversidad remarcando el rol que asumen los actores locales (Arocena, 1994), el tipo de interacciones entre estos y las características del entorno de actuación como componentes clave de procesos de desarrollo (Madoery, 2005).

No se trata de un enfoque exclusivamente preocupado por la acumulación del capital, sino también por la distribución y por las condiciones de reproducción del conjunto de la sociedad (Madoery, 2005). Es decir, el desarrollo no es sólo un proceso de mejora de la eficiencia económica, sino también de la equidad social y del equilibrio territorial y ambiental (Vázquez Barquero, 1999).

En este marco, la noción de desarrollo local también ha cambiado, transitando desde una noción de desarrollo como algo adquirido, hacia una concepción de desarrollo como algo generado a partir de las capacidades endógenas (Madoery, 2008); y desde una concepción centrada exclusivamente en lo local hacia la búsqueda de ámbitos de articulación regional. 
Este debe ser visto como un proceso, entendido como el conjunto de políticas impulsadas por los actores locales de un territorio que, sobre la base de un proyecto colectivo buscan aprovechar, bajo condiciones de sustentabilidad, las potencialidades de un territorio impactando positivamente en la economía y la sociedad, con la finalidad de satisfacer las necesidades humanas fundamentales y mejorar la calidad de vida de la población.

En este marco, el ordenamiento territorial, también adquiere nuevos roles pasando de una visión espacialista a una integradora, debe potenciar no sólo los recursos locales en el sentido tradicional (la ubicación geográfica, los recursos naturales, el ambiente, las infraestructuras, las empresas, los recursos científicos) sino que debe contribuir a hacer converger objetivos, a veces divergentes, de la totalidad de las políticas sectoriales, para poder contribuir al desarrollo a largo plazo.

De aquí que en el análisis interese reconocer articuladamente las políticas vinculadas al ordenamiento en relación a los procedimientos institucionales para lograrlo, y en su valoración respecto de los recursos del territorio, enfatizando su articulación con acciones propias del desarrollo local.

Los procedimientos institucionales construidos en este sentido deberían observarse en la complejidad adoptada por la estructura administrativa, que posibilite una estrecha articulación a nivel regional, provincial y nacional y sectorial (en la formulación, implementación, adecuación, etc.); la participación comunitaria en el proyecto de desarrollo (reconocimiento de las necesidades, recursos y capacidades preexistentes, etc.); y la capacidad de innovación tanto del gobierno local, como de las empresas e instituciones.

Por su parte se entiende que la valoración de los recursos del territorio debe considerar una visión del territorio amplia, inclusiva de lo rural y lo urbano, local y regional, y fundamentalmente como síntesis de lo socioeconómico, lo político institucional; y la construcción y reconstrucción de los recursos locales (actividades, físicos, identitarios, culturales, paisajísticos, humanos, etc.) para transformarlos en capacidades endógenas ${ }^{7}$ a partir de su puesta en valor y de la formulación de estrategias e instrumentos para lograrlo.

\footnotetext{
${ }^{7}$ En el sentido que le confiere Madoery (2008), de cualidades y dinámicas relacionales que expresan las personas, las organizaciones y las empresas de un territorio local, de cuya combinación sinérgica surgen atributos específicos que le otorgan a un territorio local mejores posibilidades de desarrollo.
} 


\section{GOBIERNOS LOCALES: DESARROLLO y ORDENAMIENTO TERRITORIAL EN LA PROVINCIA DE BUENOS AIRES DESDE 1977}

Los municipios argentinos han tenido tradicionalmente un rol marginal en cuanto a sus incumbencias para el desarrollo ${ }^{8}$, producto de un esquema institucional centralista, que ha concentrado mayor responsabilidad y recursos en las esferas administrativas nacional y provincial, otorgando a los municipios funciones limitadas a la obra pública, la provisión de servicios y la regulación urbana.

Las políticas de los gobiernos locales para impulsar la economía en su ámbito territorial, mayormente se sustentaron con políticas de inversión en infraestructuras de servicios o para la construcción de parques industriales, funciones que estuvieron vinculadas a la planificación física (Geddes, 1992) del desarrollo. De este modo se intentaba preparar el soporte físico del territorio para atraer actividades industriales buscando contribuir a la reactivación de la economía y resolver las demandas de empleo de la población de la jurisdicción.

En tanto que las políticas de ordenamiento del territorio recién han adquirido cierta aceptación en los ámbitos locales a partir de la década del '60 con el auge del "planeamiento", acogimiento que obtuvo diverso grado, tanto por procesos históricos particulares, como por el relativo desarrollo institucional de cada municipio.

A continuación se tratará de recorrer el camino experimentado por ambos tipos de políticas desde un momento de quiebre histórico a nivel nacional en lo económico, político y social, como para la provincia de Buenos Aires en lo respectivo al ordenamiento de su territorio ${ }^{9}$ cuando se instala el sistema provincial de planeamiento territorial y urbano.

\subsection{Enfoques y prácticas 1977-2001}

Para iniciar el análisis de cada periodo se los ha caracterizado brevemente a partir de reconocer: los principales rasgos de su modelo socioeconómico y político; las discusiones teóricas contemporáneas; y las políticas formuladas y/o implementadas, a partir de reconocer

\footnotetext{
${ }^{8}$ Si bien es cierto que el conjunto de municipios argentinos está caracterizado por ser un conjunto heterogéneo de realidades municipales en cuanto a formas institucionales, características geográficas, población, estructuras productivas, organización administrativa y prácticas políticas (Iturburu, 2000).

${ }_{9}$ Incluso a nivel nacional, la ley de Usos del Suelo y Ordenamiento Territorial fue la primera norma en la materia.
} 
sus enfoques, y principales estrategias valorando específicamente los procedimientos institucionales y la valoración de los recursos del territorio, siempre con énfasis en la existencia o no, de articulación.

\section{Período 1977- 1983}

El proyecto político que inició el gobierno militar durante el segundo lustro de la década del '70 implicó profundos cambios sociales y económicos que condicionaron tanto a las políticas como a la discusión en torno al modelo de desarrollo y las políticas territoriales.

Como iniciador del modelo neoliberal ${ }^{10}$, sus objetivos fueron la liberalización de los mercados, la apertura de la economía, la libre operación de los mercados de capitales, y la eliminación de los privilegios fiscales, por lo que se dio implícitamente por terminada la industrialización como objetivo de la política económica (Girbal - Blacha y otros, 2001); en tanto que en lo social implicó un momento de quebrantamiento de los derechos, por lo que las discusiones en torno a las políticas se minimizaron y limitaron en sus contenidos.

Las discusiones teóricas, en los ámbitos académicos y de investigación científica y gubernamental estuvieron vedadas, los avances teóricos y metodológicos fueron mínimos, siendo los principales temas vigentes los que surgieron de discusiones precedentes.

Paradójicamente en el mismo periodo: se decreta el principal instrumento de planificación territorial provincial, que se constituyó desde ese momento en la única política territorial para toda la provincia con continuidad, aún vigente; y se destaca también por la formulación de políticas provinciales de desarrollo, aunque con escasa implementación efectiva. Esto puede explicarse como corolario de la valorización de la planificación ${ }^{11}$ que forjaron los equipos técnicos que precedieron al mencionado periodo, vinculadas también con el desarrollismo y la descentralización territorial, como formas de resolución de los desequilibrios regionales, defendidas por distintas asociaciones profesionales y organismos de gobierno. Estas inquietudes que anteceden, junto con los análisis y estadísticas de la problemática del área metropolitana y del resto del territorio provincial producidos en los

\footnotetext{
${ }^{10}$ El modelo territorial resultante tiene como pauta predominante la integración al mercado mundial a través del proceso de globalización.

${ }^{11}$ Que había propiciado la creación de diversos organismos de planificación del desarrollo a nivel nacional y provincial, y de un organismo vinculado al ordenamiento provincial (Dirección de Ordenamiento Urbano de la Subsecretaría de Vivienda y Urbanismo) desde el año 1969.
} 
organismos de gobierno sirvieron para caracterizar el espacio y las necesidades de atención diferenciada.

De aquí que la planificación del desarrollo durante el gobierno militar, fuera diseñada desde el enfoque de la planificación regional y con un importante sesgo economicista, llegando a delimitar regiones y ejes de desarrollo, tanto a nivel nacional como provincial. En la provincia de Buenos Aires, la Secretaría de Planificación del Desarrollo (SEPLADE) -a partir de considerar al territorio provincial como un sistema de ciudades, áreas rurales, depósitos de recursos naturales y redes de transporte y comunicación- propuso en 1979 una regionalización, cuyo objetivo era instrumentar el planeamiento del desarrollo provincial. Para este estudio la región fue definida como el marco geográfico de atracción de una ciudad importante en la que se concentran las actividades económicas, sociales y culturales (Hernández, 1996), que actuaban como polos principales alrededor de los que se organizaba un conjunto de partidos menores que necesitan revitalización para los que se establecieron "estrategias de desarrollo selectivo".

A su vez, las regiones estaban agrupadas en ejes de desarrollo ${ }^{12}$ (pampeano, mediterráneo y metropolitano), cuyo funcionamiento consideraba roles complementarios, dentro de los cuales, se delimitaron microrregiones a partir de grandes ciudades ${ }^{13}$ Estas estrategias especiales estaban dirigidas a minimizar la pérdida de población urbana del interior provincial a partir del impulso de complejos agroindustriales, entendidos como “sectores de punta" de la economía provincial, capaces de dinamizar la economía en su conjunto.

La regionalización hecha por la SEPLADE ha recibido fuertes críticas, tanto por aspectos metodológicos por la falta de correspondencia entre el concepto de eje de desarrollo en su sentido clásico y la propuesta de la $\operatorname{SEPLADE}^{14}$ (Hernández, op. Cit.), y por

12 Este análisis estuvo basado en: a) la distribución de las ciudades según su población; b) la especialización funcional de las ciudades, considerando los factores industria, comercio, infraestructura y equipamiento; c) la determinación de áreas de influencia a través del análisis de flujos, medidos para las actividades industrial y comercial, y los servicios de educación y salud. Estas áreas se asimilaron a los límites de los partidos, por razones operativas.

${ }^{13}$ Es decir, se aplicaron dos tipos de delimitación territorial. Uno referido a la identificación de ejes de desarrollo y otro concerniente a la partición del territorio en función de un conjunto de ciudades ordenadas jerárquicamente (Hernández, 1996).

${ }^{14}$ En el trabajo de SEPLADE se da una versión del concepto eje de desarrollo que difiere del acuñado por Poitier, no se identifican los ejes de comunicación entre centros en los que los factores de impulso y propagación unidos a los cambios tecnológicos hayan producido la transformación de los mismos en ejes de desarrollo, y se desconocen entonces, ciertos ejes de comunicación y desarrollo que caracterizan a otras regiones que no fueron reconocidas (Hernández, 1996). 
incompatibilidad teórica y técnica en la utilización de los principales conceptos teóricos “ventajas comparativas“ y "equilibrio" 15 (Hernández, op. Cit.), por la limitación en cuanto a la definición de los factores de desarrollo, por la falta de respuestas operativas a los problemas; y por la falta de participación.

En lo que respecta al ordenamiento del territorio, la sanción del Decreto Ley 8912 estuvo urgida por la necesidad de contener el irracional proceso de subdivisión de tierras sin infraestructuras y en situaciones de riesgo que venía efectuándose en la provincia, por lo que las principales acciones de la ley tuvieron como objetivos la limitación de la subdivisión y la reducción de los factores de ocupación del suelo. De aquí que desconozca a la planificación de las áreas rurales y utilice como principal instrumento al zoning, que implica mayormente una guía para la acción privada pero no para el accionar del Estado sobre el territorio.

Sin embargo la norma introduce dimensiones e instrumentos que hasta ese momento no estaban incorporados en las prácticas de las políticas territoriales, entre ellos la noción de proceso de planificación, su visión integradora de las estrategias de desarrollo económico, social y físico, y la aparición de figuras urbanísticas innovadoras ${ }^{16}$.

Si bien las políticas de desarrollo mencionadas y la sanción de la Ley 8912 son contemporáneas, y puede afirmarse que resultan de preocupaciones confluyentes en su germen ideológico, no han tenido ninguna relación, tanto en la formulación como en la implementación efectiva. Puede inferirse que los funcionamientos de los organismos encargados de su implementación como estructuras paralelas han dificultado la articulación (más allá de ciertos procedimientos puntuales realizados en conjunto), cuestión que puede explicarse también por rencillas y prejuicios entre áreas según lo expresado en las entrevistas.

Hacia el fin de este periodo se produce el ocaso definitivo de las políticas de desarrollo frente al avance extensivo del modelo neoliberal, aunque efectivamente nunca llegaron a ser implementadas, dejando sólo un discurso ideológico (Galilea, 1983) manifestado en la

15 Hernández (Op. Cit.) hace referencia a esta incompatibilidad entre teorías al señalar que en términos analíticos, se fundamenta la separación de los ejes mediterráneo y pampeano sobre base del criterio de las ventajas comparativas. Sin embargo, no se explicita la forma en que este criterio opera en el proceso de separación territorial. También recalca Si el eje de desarrollo forma parte de los conceptos que abren paso a las teorías que explican procesos desequilibrados y las ventajas comparativas se definen dentro del contexto teórico de la teoría del equilibrio, surgiría así, una incompatibilidad teórica y técnica cuando se relacionan ambos conceptos.

${ }^{16}$ Crea mecanismos de movilización del suelo urbano que no tienen precedentes en el país: la declaración de zonas de provisión prioritaria de servicios y equipamiento; las zonas de edificación necesaria o "forzosa, y la facultad municipal de proceder a la reconformación parcelaria mediante declaración de utilidad pública y expropiación; y prevé la elaboración de planes intermunicipales (Suarez y Sarrahil, 1978). 
abundancia de publicaciones editadas por la SEPLADE, en tanto que la sanción del Decreto Ley 8912, sí comenzaba a implementarse a partir de la concreción de delimitaciones preliminares de áreas, en algunos casos a partir de planes existentes.

En síntesis, durante la primera etapa de implementación del Decreto Ley 8912, las políticas de desarrollo y ordenamiento recorrieron por sendas paralelas y han recogido escasos resultados. Su causa principal, indudablemente ha sido el sesgo autoritario del gobierno y el modelo económico instaurado, que inauguraron el largo ocaso de la planificación y la desjerarquización del Estado y las políticas públicas, incluso en contraposición con algunos de los fundamentos de los principales instrumentos formulados durante el periodo. Las principales estrategias no avanzaron mas allá de procedimientos institucionales burocratizados, si bien varios instrumentos aún hoy serían innovadores -la regionalización, la movilización del suelo urbano, etc.- escasas veces fueron implementados o considerados. En tanto que la valoración del territorio desconoció la diversidad de los recursos (urbanos, rurales, humanos, físicos, políticos, institucionales) enfatizando prioritariamente las áreas urbanas y la dimensión económica, y aunque débil en lo propositivo la regionalización en cierto modo reconoce las disparidades entre regiones.

\section{Período 1983- 1991}

En 1983, la recuperación de la democracia por parte de la sociedad argentina reintroduce en el debate -tanto en el campo político como en el de las ciencias sociales y la investigación- temáticas referidas a la formulación de políticas, planificación, descentralización, y participación (Findling y Tamargo, 1994), como valores propios del proyecto de profundización democrática. Sin embargo, las dificultades económicas del país, marcaron las prioridades y limitaciones de las políticas territoriales y de desarrollo durante casi una década.

Las nuevas visiones, empapadas del espíritu democrático, amplían las miradas espacialistas y economicistas que tenían las políticas anteriores, buscando integrar actores y visiones sectoriales, y alejarse definitivamente de las características de la planificación normativa. La descentralización es vista por su virtud democratizadora en cuanto busca aproximar las decisiones de política a jurisdicciones subnacionales y favorecería una mayor y 
más efectiva participación de los actores interesados, información, fiscalización social de la gestión pública, etc. (Vilas, 2003).

Tanto a nivel del ordenamiento, como en el resto de las políticas, la reestructuración no se produce de un día para el otro, y es hacia mediados del periodo cuando se producen los hechos de mayor relevancia.

Enmarcando las acciones del gobierno provincial para el periodo 1989-91 se formuló el Plan Trienal de la Provincia de Buenos Aires que desde su concepción también buscaba reivindicar a la planificación como un instrumento al servicio del gobierno, la participación comunitaria, el consenso político, y la concertación económica.

Dentro del Plan se concretó un trabajo de delimitación regional, que reconoce siete regiones para las que se definieron las estrategias de desarrollo ${ }^{17}, \operatorname{programas}^{18}$ y proyectos $^{19}$ concretos de gobierno, de mediano y largo plazo. Si bien la asignación de instrumentos estaba compartimentada por áreas de gobierno, el plan tuvo un enfoque interdisciplinario, con una fuerte impronta económica y presupuestaria con la idea de organizar el accionar estatal.

Para el sistema provincial de planeamiento este momento resultó relevante ya que se intentó un modelo participativo articulado con los municipios, integración interáreas entre organismos provinciales, ONGs, Universidades y otros entes privados (Garay S., 2005).

En lo que respecta a la elaboración de planes municipales se produjeron avances que otorgaron mayor profundidad y alcance a los instrumentos previos. Sin embargo, el mayor peso de la gestión en este periodo se concentró en la obra pública, siendo los principales resultados trabajos de escala regional con áreas de gobierno provincial y municipios ${ }^{20}$, y una intensa políticas de tierras ${ }^{21}$ en la que se integra al instituto de la vivienda y el ordenamiento territorial.

\footnotetext{
${ }^{17}$ Las estrategias se proponen dar respuesta a cinco problemas centrales identificados en el diagnóstico, por lo que son cinco proposiciones básicas: la movilización del potencial económico bonaerense, el mejoramiento de la calidad de vida, el manejo eficaz de los recursos naturales y el mejor uso del suelo rural y urbano; el fortalecimiento de la dotación de infraestructura social básica; y la transformación del Estado, profundizando su democratización a través de la descentralización.

${ }^{18}$ Se las identificó como áreas de integración intersectorial.

${ }^{19}$ Se las identificó como unidades de actuación de una única área de gobierno.

${ }^{20}$ Como los estudios realizados para la cuenca del Río Reconquista y la evaluación de impacto ambiental de la Autopista Buenos Aires La Plata.

${ }^{21}$ Entre las acciones de los programas que buscan mejorar la situación de la tierra y vivienda se destacan la producción de suelo urbano a partir de la movilización de tierras ociosas y a la regularización dominial.
} 
Sin embargo, la implementación de la política de tierras, no encontró los resultados esperados, debido principalmente a las insuficientes respuestas en relación a las extremas necesidades de la población y a las modalidades en la implementación.

El énfasis puesto en las políticas de tierras, por la gravedad de la situación social, minimizó la importancia otorgada por los municipios a los instrumentos globales de la planificación establecidos por la Ley 8912 (Clichevsky, 1991), y a la planificación integral del desarrollo. Esta política implicó también el desaliento paulatino de la política de vivienda y recibió fuertes críticas por la utilización del denominado "clientelismo político" para el acceso a la tierra.

Recapitulando, durante el segundo periodo analizado, cursando la primera década de implementación del Decreto Ley 8912, se observan avances en la articulación de las políticas de desarrollo y ordenamiento, basados en la valorización de la planificación, programación, participación y descentralización, como logros del proceso de reorganización del Estado democrático. Sin embargo, la profunda crisis económica limitó los logros y sesgó las acciones a decisiones presupuestarias y urgentes enfatizando la obra pública y la política de tierras.

Por esto, las políticas implementadas no lograron concretarse en procedimientos institucionales que las propendieran (articulación, interdisciplinariedad, descentralización política, regionalización) quedando relegados por la prioridad de la política de tierras y de infraestructuras. En tanto que la valoración del territorio, si bien se llegan a formular estrategias diferenciadas por regiones, continuó desconociendo la diversidad de los recursos (urbanos, rurales, humanos, físicos, políticos, institucionales) y dando énfasis lo urbano.

\section{Década del '90}

El primer periodo de transición a la democracia había transformado un régimen político, pero no pudo poner los basamentos de un nuevo sistema social de acumulación (Portantiero, 1987) ni mejorar el escenario social negativo, por lo que, a comienzos de la década del '90, con el segundo gobierno democrático, las políticas públicas consolidaron finalmente el perfil neoliberal que condicionó bajo su ideología, por acción o reacción, al resto de las políticas. 
Este modelo, bajo los fundamentos del "pensamiento único", donde el poder decisorio sobre la acumulación del capital y la asignación de recursos se transfiere desde los espacios nacionales a los mercados transnacionales (Ferrer, 2001), reconoce primordialmente actores globales y lógicas sistémicas irrefutables y desconoce el territorio y los actores locales, desvinculando en términos generales, la economía de los ámbitos territoriales.

La denominada "Reforma del Estado" 22 instituida en Argentina a comienzos de la década del '90, dio lugar a procesos de descentralización, privatización y ajuste estructural de la economía, aplicó un programa concebido desde una lógica "fiscalista" que transfirió competencias y funciones desde el gobierno nacional a los gobiernos provinciales y locales, recargó las agendas de estos últimos suponiendo que las fuerzas del mercado serian elementos suficientes para crear condiciones de crecimiento de las inversiones productivas. La descentralización en este nuevo contexto se diferencia mucho de los procesos de la década anterior, cuando tenía mayor relación con la participación y democratización (Villar, 2004). Los efectos han sido contrarios y la falta de atención a las dimensiones microeconómica y territorial, terminaron afectando la estabilidad macroeconómica.

La prioridad del nivel central otorgada a lo macroeconómico, marcó un nuevo escenario $^{23}$ que obligó a los municipios a pensar en el desarrollo local como una salida, redefiniendo sus roles y estrategias de intervención sin contar muchas veces con las herramientas, recursos y capacidades institucionales necesarias.

En este marco, los municipios argentinos han organizado, planificado y gestionado, en la medida de sus posibilidades, políticas locales, asumiendo el desafío de encarar los retos del desarrollo desde la especificidad, en cuanto a potencialidades y problemáticas de cada territorio, cristalizándose de diferentes modos según el contexto, replanteando los modelos tradicionales de gestión local.

\footnotetext{
${ }^{22}$ Las preocupaciones que definen la agenda de los '90 son la alta inestabilidad económica, la crisis fiscal que condicionaba la formulación de políticas y limitaba las posibilidades de actuación del Estado, la ineficiencia de la administración pública y la incapacidad del Estado para dar respuestas a los problemas centrales que afectaban a la sociedad. (Madoery, 2005)

${ }^{23}$ El nuevo escenario que condicionó fuertemente a los territorios locales estuvo marcado por los cambios en las estructuras productivas que se tradujeron en una fuerte concentración y centralización de actividades económicas (Kosacoff, 1998; Basualdo, 2000); la pérdida de peso relativo de aquellos sectores de empresas (Pymes y empresas públicas nacionales), con mayor vinculación con los territorios donde desplegaban su actividad (Katz, 2000). De la misma manera repercutieron los cambios en las formas de organización social de la producción por la desaparición de las formas propias del modo de sustitución de importaciones y el incremento de la economía informal y de subsistencia (Madoery, 2005).
} 
Surgieron así a mediados de la década los Corredores Productivos, consorcios de municipios que se unieron por necesidades comunes ${ }^{24}$ para estimular proyectos productivos, iniciativa que posteriormente fue promovida por el nivel provincial ${ }^{25}$. Esta regionalización implicó un cambio de perspectiva respecto al esquema de regionalización difundido durante los 60 y 70, ya que para diseñar la nueva organización del territorio provincial enfatiza en los flujos de circulación e intercambio, ponderando la conectividad frente a la proximidad, que fundamentaba la delimitación polarizada anterior (Gorenstein, 1998) además del cambio en lo institucional en lo que respecta al rol de los actores territoriales y el énfasis puesto en la identificación de proyectos concretos (Elgue, 2002).

Estas iniciativas han tenido resultados dispares, en su mayoría escasos o destinados a la resolución de problemas puntuales. Puede encontrarse una respuesta en los motivos que plantea Villar, uno de dimensión política (los consorcios no se constituyen en instituciones de gobierno) y el otro de modalidad de gestión (el Estado provincial carece de estructuras burocráticas preparadas para asumir una visión integrada del territorio). Y un fuerte condicionante local, lo constituye la debilidad institucional de los gobiernos municipales.

En esta época también han surgido iniciativas para crear suelo industrial en ciudades, e infraestructuras para explotar nuevos perfiles de actividad económica (por ejemplo las zonas francas), que han tenido como principal elemento condicionante del desarrollo la asignación estática de infraestructuras, restringiendo la valoración de las capacidades propias de una sociedad para llevar adelante el proceso de desarrollo. Muchas zonas industriales han sido creadas con esta perspectiva y no han resultado provechosas y no porque no se hayan asentado establecimientos.

En el mismo periodo muchos municipios han recurrido a la Planificación Estratégica para conducir procesos de desarrollo local de una ciudad o región, cuyos principales argumentos se sustentan en valorizar el componente político del proceso de planificación, la gestión social y la valorización de la acción entre otras cuestiones. Sin embargo las estrategias vinculadas al ordenamiento del territorio y ambiental pocas veces han sido retomadas y desarrolladas en planes e instrumentos territoriales (Garay S., Op. Cit), aunque fueron las áreas de planeamiento quienes mayormente los condujeron. Incluso, se confronta a la

\footnotetext{
${ }^{24}$ En 1994 Corredor del Sudoeste fue la primera asociación intermunicipal de la provincia, surgida de las propias necesidades locales de mejorar la ruta entre los municipios de General Lamadrid y Laprida.

${ }^{25}$ Impulsados por el Ministerio de la Producción y el Empleo, a través de la Subsecretaría de Industria, Comercio y Minería.
} 
planificación estratégica y a los planes territoriales y urbanos como instrumentos disociados, incluso contrapuestos. Las principales críticas hechas a la planificación estratégica hacen eco de la lectura de diagnósticos muy estructurados de antemano, que desconocen la existencia de cuestiones diferenciales entre distintos espacios territoriales, y la simplificación en la construcción de los escenarios futuros (Garay, 2004), quizás por la excesiva rigidez y simplificación metodológica.

Sin embargo se rescata como resultado positivo que estas iniciativas han servido para movilizar y estimular la participación comunitaria en los municipios, valorar recursos, delinear planes de gobierno y para construir diagnósticos precisos y actualizados sobre la situación local.

En lo que respecta a las políticas territoriales formuladas e implementadas en la Provincia, las acciones centraron sus ejes en la ejecución de obras públicas y en la política de tierras, concebidas desde un modelo de gestión sectorial donde cada área de la administración operó con objetivos particulares, competencias ambiguas y muchas veces superpuestas (Garay S., Op. Cit.). Incluso el nivel nacional avanzó sobre competencias provinciales en la ejecución de grandes obras públicas y en el manejo del endeudamiento provincial. Esto se condice con la disminución jerárquica que sufrieron las áreas vinculadas con el ordenamiento urbano a nivel provincial, fundamentalmente en el periodo 1991-2004 de la misma manera que en muchos municipios se eliminaron las áreas de planeamiento y se dedicaron a atender temas de coyuntura (Garay S., Op. Cit.).

Es decir, esta ausencia de planificación, tanto a nivel provincial como municipal, implicó indiferencia hacia las respuestas necesarias ante el profundo proceso de transformación territorial emergente, marcado por la fragmentación, privatización y dispersión territorial. En este marco, muchas jurisdicciones implementaron normas no encuadradas en la jurisdicción marco, interpretaciones sectoriales aprobadas por excepciones para casos y temas puntuales, produciendo muchas veces solo cambios normativos puntuales sin estudios técnicos en función de favorecer emprendimientos que generen empleos (Garay S., Op. Cit.). Y en lo que respecta a la profundización del proceso de planeamiento, muy pocos municipios produjeron una actualización o avances.

Es decir, la década del noventa, que ha sido un periodo de fuertes retrocesos para el desarrollo, debido al énfasis puesto por el Estado en las políticas macroeconómicas, y el desconocimiento de las dimensiones micro y territorial, también implicó que la relación entre 
desarrollo y ordenamiento a nivel nacional y provincial nunca existiera, limitando la política territorial provincial a la obra pública y a la política de tierras. Sin embargo, durante este periodo, muchos municipios en forma autónoma, y dependiendo sólo de liderazgos locales, han implementado procesos de desarrollo local con diversas estrategias, como salida ante la retirada del Estado y ante el contexto global, atendiendo a las discusiones que se venían sucediendo en ámbitos académicos nacionales y extranjeros, e incluso desde organismos internacionales.

Por esto, los procedimientos institucionales, han tenido una situación diferencial según las capacidades de los municipios; pero ha sido escaso el interés por facilitar o promover la articulación desde el nivel provincial, tanto por la escasa complejidad de las estructuras administrativas, como por la falta de institucionalidad de los instrumentos utilizados (consorcios, planes, etc.), por lo que se ha tenido una visión sectorial frente a problemas puntuales. La valoración del territorio fue desconocida, priorizando la asignación estática de recursos (infraestructuras y tierras).

\subsection{Hacia enfoques convergentes entre desarrollo y ordenamiento}

Distintas discusiones sobre el desarrollo -muchas preexistentes, y que venían influyendo en las políticas e instrumentos anteriores- hacia fines del Siglo XX, acercan alternativas luego de la profunda crisis del modelo neoliberal ocurrida en Argentina e incorporando un perfil más cercano a lo productivo, y no sólo basado en la actividad financiera que caracterizó las dos décadas anteriores (Arroyo, 2006). Desde una visión holística y constructiva del desarrollo (Boisier, 2003) se aproxima a la realidad admitiendo la diversidad, remarcando el rol que asumen los actores locales (Arocena, 1995), el tipo de interacciones entre estos y las características del entorno de actuación como componentes clave de procesos de desarrollo.

Estas propuestas, frecuentemente interdisciplinarias, consideran al territorio como la variable que sintetiza la diversidad social, económica y política del proceso de desarrollo, tanto a escala mundial, nacional y local (Benko y Lipietz, 1994; Storper, 1997; Caravaca Barroso, 1998; Boscherini y Poma, 2000; Yoguel, 2000; Santos, 2002: citados en Manzanal, 2006) 
El proceso de desarrollo local es visto entonces como el resultado del esfuerzo organizativo e innovador del conjunto de la sociedad y no sólo del correcto desempeño de los mercados, por lo que la estrategia local busca promover la dinamización empresarial, el estimulo de las innovaciones, la generación de externalidades derivadas de la asociatividad y la proximidad, y la necesidad de construir una visión común del desarrollo territorial, para la utilización eficaz de todo el potencial de desarrollo disponible e incorporable al territorio (Alburquerque, 2003). Por eso que el proceso de planificación del desarrollo local debe ser participativo, llevado a cabo por actores locales, garantizando la participación de los agentes interesados y de los afectados.

En similar sentido Vázquez Barquero (1999) refiere al desarrollo endógeno como un proceso de crecimiento económico y cambio estructural liderado por la comunidad local utilizando el potencial de un territorio que conduce a mejorar el nivel de vida de la población. Identifica tres dimensiones una económica, otra sociocultural y otra política, y destaca como factores determinantes la Innovación y difusión del conocimiento, la organización flexible de la producción, el desarrollo urbano del territorio y la densidad institucional.

De acuerdo a Madoery (2005) cobran sentido para el desarrollo los procesos productivos territorialmente vinculados a través de eslabonamientos o cadenas de valor; rasgos específicos de la cultura y del "saber hacer" local que se refuerzan como "factores de especificidad territorial" (Pecqueur y Colletis, 1995). En esta afirmación se hace hincapié en la trascendencia de la especificidad del territorio en cuanto valores incorporados a los procesos productivos a partir de los recursos humanos, culturales y organizaciones locales, y a la posibilidad de respuesta y adecuación a los cambios. Quedando implícita la idea aprovechar las ventajas originadas en el contexto y"endogeneizarlas".

Benko (1999) al referirse al desarrollo local lo define como el enfoque que propone retornar a una visión territorial del desarrollo donde se preconizan acciones por la puesta en valor de los recursos locales, con la participación de la población integrando atributos del medio (económicos, sociales, culturales y políticos). Se apoya en la visión territorial "integradora" de aspectos sociales, económicos, culturales y políticos y cercana a la posibilidad de ser instrumentada desde la planificación y el ordenamiento territorial.

Alburquerque deja traslucir esta visión integradora y su interés por el enfoque territorial, con la posibilidad de incorporar como instrumentos al ordenamiento territorial y a la planificación desde una dimensión estratégica, resultando una concepción integral que 
incorpora una perspectiva intersectorial. Integra en la visión de desarrollo aspectos económicos, sociales, medioambientales y culturales, asignando al gobierno local la posibilidad de generar el entorno (preparar el territorio) con infraestructuras y servicios necesarios para que el sector empresarial asuma el papel de productor y dinamizador de la economía local.

Es decir, el potencial de desarrollo de un territorio local no depende sólo de ventajas iniciales (como la ubicación geográfica, la riqueza del suelo o la dotación estática de recursos tales como infraestructuras, empresas, universidades) como tampoco de la inyección exógena de recursos productivos (inversiones, aportes financieros, etc.), sino que es necesario transformarlos en capacidades endógenas (Madoery, 2008) a partir de un conjunto de acciones estratégicas articuladas.

En consonancia con esta visión, el ordenamiento territorial -tanto como disciplina científica, como técnica administrativa y como política- es fundamental por su contenido planificador, en tanto expresa y articula el resultado de las políticas económicas, sociales, culturales y ecológicas, compatibilizándolas con el equilibrio intra e interregional, la organización física del espacio, la utilización racional de los recursos naturales y la conservación ambiental.

Por cuanto que, el ordenamiento territorial debe potenciar no sólo los recursos locales en el sentido tradicional (los recursos naturales, el tipo de suelo, el ambiente, las infraestructuras) sino que debe contribuir a hacer converger objetivos, a veces divergentes, de la totalidad de las políticas sectoriales, y particularmente de la de desarrollo local.

Esta necesidad de "articular" y la idea de transversalidad de ambas políticas, se verifica en la convergencia de los fundamentos teóricos contemporáneos de ambos enfoques, en los que Chabalgoiti (2004) y Madoery (2008) coinciden: carácter procesual, complejidad y diversidad $^{26}$.

Procesual, en tanto que las transformaciones reconocen cada proceso histórico sobre el que las alternativas posibles se adecuan a cada realidad territorial, pero éstas son "creadas" a través de un proyecto en el cual la acción planificada cumple un rol fundamental. Complejidad en el sentido que es imprescindible reconocer la multidimensionalidad y

26 Exactamente Chabalgoity señala como cuestiones clave a considerar en la elaboración de marcos conceptuales, políticos e instrumentales para la planificación y gestión del territorio el carácter procesual y la complejidad; en tanto que Madoery apunta como rasgos estructurales en el sustento de un proyecto de desarrollo endógeno, a la complejidad, la diversidad y la estrategia. 
dinámica de cada territorio, involucrando aspectos socioeconómicos, políticos, institucionales, culturales, y físicos. Y Diversidad, en tanto que las características y capacidades son específicas de cada territorio.

\section{Prácticas recientes}

Tanto los aportes recientes vinculados al desarrollo local, como los anteriores, han repercutido en las políticas formuladas e implementadas con posterioridad a la crisis de 2001 en Argentina. Esto puede observarse al hacer una lectura de los numerosos instrumentos nacionales y provinciales que buscan fomentar el desarrollo local ${ }^{27}$, sin embargo, aun queda pendiente la valoración del rol del ordenamiento territorial en su verdadera magnitud.

Sin embargo en su formulación se verifica cierto centralismo, fundamentalmente a partir de la disponibilidad de recursos que permite a la nación ofertar mayor cantidad de programas, y a la tradicional postura de los municipios como demandantes de los niveles superiores. No existen instrumentos en instancias intermedias que reconozcan especificidades de las regiones y territorios locales, considerando que lo nacional y lo local forman parte de un mismo proyecto de desarrollo, sin ignorar la capacidad de coordinación de los niveles superiores pero valorando potenciales específicos locales (Ríos y Rocca, Op. Cit.).

Estas propuestas que buscan el desarrollo local parecen desarticular sus distintas dimensiones según los organismos de gobierno que las formulen, no son integrales y plantean en general el mejoramiento de la capacidad emprendedora en sus sistemas económicos y en fortalecer las economías sociales. Sólo se observa una articulación de lo económico con lo social sólo en los programas que están destinados a mejorar la situación de sectores desfavorecidos social y económicamente, y con programas que, si bien se alejan de las políticas asistencialistas, están orientados a la promoción no integral del desarrollo productivo y social. Se entiende que deberían complementarse con otras que promuevan el desarrollo productivo en otras escalas, no solo regulando actividades, y articulándolas con las políticas sociales para que los cambios no se manifiesten solo en el empleo y en la producción, y

\footnotetext{
${ }^{27}$ Pueden verse en el trabajo "El Desarrollo Local y su Relación con el Ordenamiento Territorial" (RÍOS Y ROCCA, 2007), en el que se observa la coordinación de experiencias actuales (nacionales y provinciales) que buscan favorecer el desarrollo local con estrategias de ordenamiento.
} 
puedan construirse proyectos de corto plazo (para las situaciones críticas) y otros de mediano y largo plazo (Ríos y Rocca, Op. Cit.).

Si bien el rol del territorio aparece implícito en el desarrollo local, porque es el que sustenta por definición a las políticas, en éste no se ha pensado la manera de planificar el ordenamiento para construir un territorio competitivo. Como tampoco la manera de planificar el territorio según las situaciones diferenciales de los municipios (presupuesto, capacitación, protagonismo, relaciones entre municipios, contexto) e incluyendo la gestión del proceso (Ríos y Rocca, Op. Cit.).

Este es el panorama que configuran los instrumentos que buscan fomentar el desarrollo local desde organismos de gobierno nacionales y provinciales.

De igual forma que en el periodo anterior, se identificó un conjunto de iniciativas puntuales de desarrollo local, que indican cierta maduración, al considerar un territorio de gestión más amplio que el municipal, e incorporar la perspectiva regional, como las asociaciones entre Olavarría, Tandil y Azul; Bahía Blanca y Coronel Rosales; Plan Estratégico Tuyú Mar y Campo; Región Litoral -Villa Constitución, San Nicolás; Ramallo y San Pedro- (Madoery, Op. Cit).

Se ha producido una revalorización del ordenamiento territorial en la estructura administrativa provincial $^{28}$ y nacional que busca una correspondencia entre teoría y metodología impulsando la planificación territorial provincial. Sin embargo, los resultados hasta ahora alcanzados han sido escasos, con avances en cuanto a la realización de diagnósticos y formulación de estrategias sólo para el Área Metropolitana de Buenos Aires. Todavía no se han enunciado los Objetivos y Estrategias para el Ordenamiento Provincial requeridos por el Decreto Ley 8912 para dar marco a los planes de escala territorial, regionales y locales.

Muchos municipios del interior provincial, evidencian cierta debilidad en la lectura de sus organigramas, por poseer estructuras elementales y poco integradoras. Dependiendo entonces de la voluntad y conveniencia política la integración de niveles y sectores, y particularmente del liderazgo de intendentes, secretarios y subsecretarios, el ingreso de la cuestión territorial en la agenda de gobierno.

\footnotetext{
${ }^{28}$ Reestableciendo la Subsecretaría de Urbanismo y Vivienda dentro del Ministerio de Infraestructura, Vivienda y Servicios Públicos que había sido disuelta en 1995.
} 
Esto se demuestra al analizar el estado del proceso de planificación municipal desde la sanción del Decreto Ley 8912, donde se observa que su evolución ha sido mínima. Particularmente, un análisis panorámico de la situación de los municipios pequeños y medianos del interior provincial, se evidencia que en mayor número no han superado la instancia de Delimitación Preliminar de Áreas.

En síntesis, pasados más de treinta años de la sanción del Decreto Ley 8912 y recorrido veinticinco años de consolidación del Estado democrático, aún hoy, en términos generales el ordenamiento territorial, y sus instrumentos, no son valorados por el Estado provincial como herramientas para impulsar el desarrollo, restringiendo todavía su visión al uso de factores de ocupación del suelo, usos y densidades, e inclusivo sólo de las áreas urbanas. Sin embargo la cantidad de instrumentos, aunque sectoriales, mayormente vinculados al desarrollo de lo económico, implican los pasos iniciales hacia etapas de mayor articulación y profundidad de las políticas.

En lo que hace a los procedimientos institucionales se denota una diferencial situación del desarrollo local, que ha entrado en la agenda del gobierno provincial, evidenciada en la cantidad de instrumentos identificados, y en la cantidad de organismos que buscan confluir hacia el mismo, y una escasa valorización del ordenamiento territorial como instrumento para lograr ese desarrollo.

Por lo que la valoración de los recursos del territorio denota esa visión del territorio, que jerarquiza aspectos económicos o sociales, y da preponderancia a los grandes centros urbanos y metropolitanos, siendo casos aislados.

\section{CONSIDERACIONES FINALES}

Se vieron las coincidencias en los fundamentos teóricos contemporáneos entre ordenamiento territorial y desarrollo local, rescatando el carácter procesual, la complejidad, y la diversidad de ambas políticas.

El fin de la articulación de las políticas de desarrollo local con las acciones propias del ordenamiento del territorio se apoya en la doble intención de generar el "territorio adecuado" para facilitar la real implementación de las primeras, a la vez que estas no conduzcan a acciones que menoscaben las potencialidades del territorio. 
Es decir, los valores que incorpora la consideración articulada ordenamiento del territorio - políticas de desarrollo, están relacionados con la adecuación de los entornos territoriales para contribuir a "endogeneizar" el desarrollo, ya sea proveyendo de las infraestructuras necesarias para, la competitividad de las empresas, la preservación del entorno ambiental, el atractivo territorial para las inversiones, etc., pero sobre todo para mejorar la calidad de vida de la población.

Por esto será importante la articulación entre todos los actores del territorio, donde el municipio sea conductor, y la sociedad participa en forma igualmente activa. En tanto que el ámbito ideal de definición de las políticas no debe necesariamente coincidir con los límites municipales, sino que podrá ser determinado por la trama de actores e instituciones, los recursos del territorio, las acciones de gobierno y las relaciones con el entorno exterior.

Por su parte, de la constatación empírica en el ámbito de la provincia de Buenos Aires, surge que la integración de políticas de desarrollo y ordenamiento fue valorada en contadas ocasiones. En general ha existido una excesiva confianza en idealizar algún tipo de instrumentos (regionalizaciones, estrategias, obra pública, planificación estratégica, normas) asumiendo que por sí solos resolverían los problemas de los espacios locales; sumado a que las políticas de desarrollo y ordenamiento han sido generalmente formuladas e implementados desde ámbitos compartimentados y supralocales, y con objetivos guiados por la gravedad de la situación de la población más pobre.

Con posterioridad a la crisis de 2001 en Argentina se ha abierto una ventana de posibilidades para la cuestión local, donde pareciera que aún no se ha valorado al ordenamiento como instrumento fundamental para aprovechar el potencial de los territorios. En el caso de las propuestas de los niveles nacional y provincial, en general no reflejan cambios en los hechos concretos (Ríos y Rocca, 2007) ya que se verifica centralismo, desconocimiento de situaciones diferenciales, desarticulación en dimensiones de actuación y falta de valoración de la planificación del ordenamiento para construir un territorio competitivo,

Si bien desde las posibilidades que otorga el marco político institucional de los municipios de la Provincia, existen pocas condiciones para que los gobiernos de los municipios pequeños y medianos del interior provincial puedan alcanzar un rol protagónico en el desarrollo local, y lo hecho hasta el momento no ha tenido resultados positivos concretos, el nuevo contexto puede entenderse como de transición hacia instancias de mayor 
valoración de los territorios locales a partir de una mejor articulación de las políticas de ordenamiento con las propuestas para su desarrollo.

\section{BIBLIOGRAFÍA}

ALBURQUERQUE, F. (2003): Teoría y Practica del Enfoque del Desarrollo Local. Artículo de la Consultoría en capacitación del Programa Mas Región, UE, Coquimbo, Chile.

AROCENA, J., (1994): El Desarrollo Local. Un desafío contemporáneo. CLAEH, Universidad Católica del Uruguay, Ed. Nueva Sociedad, Caracas.

ALMEIDA CURTH, Daniel (1978): Artículo Ley 8912: Esquema del Proceso de Elaboración en Espacios CEPA No 17. Buenos Aires, Espacio Editora.

BENKO, George (1999): La Ciencia Regional, Ed. Universidad Nacional del Sur, Bahía Blanca.

BOISIER, Sergio (2001): Desarrollo Local, ¿De qué Estamos Hablando? En "Transformaciones globales, instituciones globales, y políticas de desarrollo local", A.Vazquez Barquero y Oscar Madoery (compiladores). Homo Sapiens Editores, Rosario.

BOISIER, Sergio (1997): Vuelo de una Cometa. UnaMetáfora para una Teoría del DesarrolloTerritorial, Documento No 97/37. ILPES CEPAL Santiago de Chile.1997

BONO y otros (2004): Problemáticas territoriales, vulnerabilidad y modalidades de intervención. Publicación (CD) de las Jornadas de Investigación 2004. Facultad de Arquitectura y Urbanismo. UNLP

BOSCHERINI, F. y POMA, L. (2000), Territorio, conocimiento y competitividad de las empresas, El rol de las instituciones en el espacio global, Miño y Dávila Editores, Bs. As. Citado en Madoery, 2005.

CHABALGOITY, Miguel (2004): La Ordenación del Territorio: Aportes a un Debate Nacional. V Coloquio sobre Transformaciones Territoriales: Nuevas Visiones en el Inicio del Siglo XXI. La Plata.

CLICHEVSKY, Nora (1991): La Planificación Urbana Posible en los Ochenta. En revista Ciudad y Territorio $N^{o}$ 86-87, otoño- invierno 4/90 -1/91. Instituto Nacional de la Administración Pública, Madrid.

ELGUE, Mario César y HADDAD Verónica (2002): Consorcios productivos bonaerenses: Descentralización territorial y cooperación intermunicipal. Magazine Desarrollo Humano e Institucional en América Latina, Edición, $\mathrm{N}^{\mathrm{o}} 30$. http://www.iigov.org/dhial/dh30/dhial30_05.htm

FERRER, Aldo (2000): El Capitalismo Mágico, Diario Clarín, artículo de opinión. Bs. As., $6 / 2000$.

FINDLING Liliana y TAMARGO, María del Carmen (1994): Planificación, Descentralización y Participación: Revisión y Crítica. CEAL - UBA. Buenos Aires. 
GEDDES, M (1992): The sectorial approach to local economic policy. Citado en Da Rosa Pires, Op. Cit.

GALILEA (1983): La Enseñanza de la Planificación Territorial en América Latina: Escenarios, Problemáticas y Perspectivas. En Revista Interamericana de Planificación, S.I.A.P., Vol. XVII, nº 67, México, Sept. 1983, 26.

GARAY, Alfredo (2004): Revisión crítica de los planes estratégicos desarrollados en Argentina durante la década de los noventas:

GARAY, Susana (2005): Formulación de Lineamientos Estratégicos para el Territorio Metropolitano de Bs. As.: Subsistema de Ordenamiento Urbano y Territorial. Etapa de Diagnóstico 2005.Dirección Provincial de Ordenamiento Urbano yTerritorial. Subsecretaría de Urbanismo y Vivienda. MIVSPU.

GIRBAL - BLACHA, Noemí (coordinadora) y otros (2001): Estado, Sociedad, y Economía en la Argentina: 1930 - 1997. Ediciones, Universidad Nacional de Quilmes.

GOMEZ OREA, Domingo (2001): Ordenación Territorial. Ediciones Mundi Prensa, Madrid.

GORENSTEIN, Silvia (1998): Evolución y Perspectivas de los Consorcios Productivos Bonaerenses. En "Consorcios Productivos Municipales ", Cuadernos No4 del IPAP, Serie Investigación.

HERNANDEZ, Ruby Daniel (1996): Un Modelo de Desarrollo Regional, Grupo Banco de la Provincia de Buenos Aires y editado por Ediciones Machi

ITURBURU, M. (2001): Municipios Argentinos. Potestades y Restricciones Constitucionales para un Nuevo Modelo de Gestión Local. INAP, Buenos Aires.

KOZLAREK, O. (2001): Globalización y la Pluralidad de las Teorías Normativas. Seminario dictado en UBA. Citado en Madoery, 2005,Op. Cit.

MADOERY, Oscar (2008): Otro Desarrollo: El cambio desde las ciudades y regiones. Colección Ciencias Sociales, Serie Desarrollo y Territorio. Ediciones UNSAM.

(2005): La Primera Generación de Políticas Locales de Desarrollo en Argentina: Contexto, Características y Desafíos. Revista Política y Gestión, Vol. 8, Año 2005, Homo Sapiens Editores, Rosario.

MANZANAL, M., NEIMAN. G. Y LATUADA. M. (2006): Desarrollo Rural: Organizaciones, Instituciones y Territorios. Ediciones CICCUS

PORTANTIERO, Juan Carlos (1987): “La Transición: entre la Confrontación y el Acuerdo”, en Nun J. y Portantiero, J., Ensayos sobre la Transición democrática en la Argentina, Puntosur, Bs. As., 1987. Citado en GIRBAL BLACHA, 2001.

RIOS, Licia y ROCCA, María J. (2007): Desarrollo Local y su Relación con el Ordenamiento Territorial. Actas de "IV Seminario Internacional de Ordenamiento Territorial". CIFOTUNCUYO, Mendoza.

SUAREZ, Odilia y SARRAHIL, Eduardo (1978): Artículo Un Paso Trascendente hacia la Planificación Urbana en Espacios CEPA N ${ }^{0} 17$. Buenos Aires, Espacio Editora. 
VILLAR, A (2004): Una década de desarrollo local en Argentina. Balance y perspectivas, En Revista Electrónica "Mundo Urbano N No 24", www.mundourbano.unq.edu.ar

VILAS, Carlos (2003): Descentralización de Políticas Públicas: Argentina en la Década de 1990. Instituto Nacional de Administración Pública, Dirección de Estudios de Información.

(Recebido em outubro/08. Aceito em fevereiro/09) 